\title{
Electron microscopy and spectroscopic study of structural changes, electronic properties and conductivity in annealed $\mathrm{Li}_{x} \mathrm{CoO}_{2}$
}

\author{
Halyna Volkova ${ }^{a}$, Kevin Pachuta $^{b},{\text { Kyle } \text { Crowley }^{c}, \text { Santosh Kumar Radha }}^{c}$, Emily Pentzer $^{d}$, \\ Xuan P.A. Gao ${ }^{c}$, Walter R. L. Lambrecht ${ }^{c}$, Alp Sehirlioglu ${ }^{b}$, Marie-Hélène Berger ${ }^{a}$ \\ a MINES Paris, PSL University, Centre des Matériaux, \\ CNRS UMR 7633, BP 8791003 Evry Cedex, France \\ ${ }^{b}$ Department of Materials Science and Engineering, \\ Case Western Reserve University, Cleveland, Ohio 44106-7204, USA \\ ${ }^{c}$ Department of Physics, Case Western Reserve University, Cleveland, Ohio 44106-7079, USA and \\ ${ }^{d}$ Texas A\&M University, College Station, TX 77843-3003
}

\begin{abstract}
Chemically exfoliated nanoscale few-layer thin $\mathrm{Li}_{x} \mathrm{CoO}_{2}$ samples are studied as function of annealing at various temperatures, using transmission electron microscopy (TEM) and Electron Energy Loss Spectroscopies (EELS) in various energy ranges, probing the O- $K$ and Co- $L_{2,3}$ spectra as well as low energy interband transitions. These spectra are compared with first-principles density functional theory (DFT) calculations. A gradual disordering of the $\mathrm{Li}$ and Co cations in the lattice is observed starting from a slight distortion of the pure $\mathrm{LiCoO}_{2} R \overline{3} m$ to $C 2 / m$ due to the lower $\mathrm{Li}$ content, followed by a $P 2 / \mathrm{m}$ phase forming at $\sim 200^{\circ} \mathrm{C}$ indicative of Li-vacancy ordering, formation of a spinel type $F d \overline{3} \mathrm{~m}$ phase around $250^{\circ} \mathrm{C}$ and ultimately a rocksalt type $F m \overline{3} \mathrm{~m}$ phase above $350^{\circ} \mathrm{C}$. This disordering leads to a lowering of the band gap as established by low energy EELS. The Co- $L_{2,3}$ spectra indicate a change of average Co-valence from an initial value of about 3.5 consistent with Li-deficiency related $\mathrm{Co}^{4+}$, down to 2.8 and 2.4 in the $F d \overline{3} m$ and $F m \overline{3} m$, indicative of the increasing presence of $\mathrm{Co}^{2+}$ in the higher temperature phases. The $\mathrm{O}-K$ spectra of the rocksalt phase are only reproduced by a calculation for pure $\mathrm{CoO}$ and not for a model with random distribution of $\mathrm{Li}$ and Co. This indicates that there may be a loss of Li from the rocksalt regions of the sample at these higher temperatures. The conductivity measurements indicate a gradual drop in conductivity above $200^{\circ} \mathrm{C}$. This loss in conductivity is clearly related to the more Li-Co interdiffused phases, in which a low-spin electronic structure is no longer valid and stronger correlation effects are expected. Calculations for these phases are based on DFT $+U$ with Hubbard-U terms with a random distribution of magnetic moment orientations, which lead to a gap even in the paramagnetic phase of $\mathrm{CoO}$.
\end{abstract}

\section{INTRODUCTION}

The discovery of graphene and its associated ultrahigh electron mobility ${ }^{112}$ led to interest in fundamental and applied research on other two-dimensional (2D) atomically thin materials, such as transition metal dichalcogenides, $\stackrel{3,4}{, 4}$ black phosphorus, $\frac{5}{5}$ antimonene $\left[\frac{6}{2 \mathrm{D}}\right.$ oxides have received relatively less attention thus far, ${ }^{7}$ although many oxides exist in layered forms which might be amenable to exfoliation. Furthermore, the formation of a two-dimensional electron gas (2DEG), the prime playing ground for high-electron mobility, has been found possible at oxide surfaces and interfaces ${ }^{811}$ Two dimensional oxides may also be expected to be more stable in extreme environments and have a multitude of functionalities, from catalysis to varying types of magnetic and ferroelectric ordering, superconductivity and metal-insulator transitions. ${ }^{12}[15$ However, their potential for high-speed ultrathin transistors has not yet been established. While some 2D oxides, such as $\mathrm{MoO}_{3}$ and $\mathrm{V}_{2} \mathrm{O}_{5}$ exhibit van der Waals bonded neutral layers and can be mechanically exfoliated, $\frac{16 \mid 17}{1}$ they require doping or intercalation to increase the conductivity. Other oxides such as $\mathrm{LiCoO}_{2}$ exhibit a layering of the two cations with alternating $\mathrm{CoO}_{2}^{-1}$ and $\mathrm{Li}^{+1}$ layers which are electrostatically bonded. Such materials can be exfoliated by a combination of chemical and mechanical exfoliation techniques to achieve nanosize few atomic layer thin flakes. While the properties of bulk $\mathrm{Li}_{x} \mathrm{CoO}_{2}$ as function of $\mathrm{Li}$ concentration have been amply studied in the context of $\mathrm{LiCoO}_{2}$ based batteries, $15|18| 19$ the properties of nanoflakes are still not well known.

Recently, a chemical exfoliation procedure was established for $\mathrm{LiCoO}_{2} \cdot{ }^{20 \mid} 22$ Using additional mechanical exfoliation it becomes possible to also study the conductive properties of such ultrathin nanoscale samples.23 However, preparing contacts to these nanoscale structures requires annealing and the properties of these materials as function of temperature therefore need to be understood. This article focuses on $\mathrm{LiCoO}_{2}$ nanoflakes with the study of their crystallographic structure, electronic structure and conductive properties. The electronic structure is probed using various forms of Electron Energy Loss Spectroscopy (EELS) and the structure and morphology are studied using Selected Area Diffraction (SAED) in a transmission electron microscope (TEM). The spectra are interpreted with various types of first-principles electronic structure calculations and trends are established as function of temperature and correlated with the structural evolution of the samples. 


\section{EXPERIMENTAL METHODS}

The $\mathrm{Li}_{0.37} \mathrm{CoO}_{2}$ nanoflakes were obtained by exfoliation in three steps. First, $\mathrm{Li}^{+}$was substituted by $\mathrm{H}^{+}$ in a $1 \mathrm{M} \mathrm{HCl}$ aqueous solution. Then, $\mathrm{H}^{+}$was replaced by larger molecules of tetramethylammonium hydroxide $\left(\mathrm{N}\left(\mathrm{CH}_{3}\right)_{4}-\mathrm{OH}, \mathrm{TMAOH}\right)$ to expand the space between $\mathrm{CoO}_{2}$ layers. The $\mathrm{H}$ associates with the $\mathrm{OH}$ group and leaves the same as $\mathrm{H}_{2} \mathrm{O}$ while the $\mathrm{TMA}^{+}$ions replace the initial $\mathrm{Li}^{+}$. Due to the increased interlayer distance, the $\mathrm{CoO}_{2}^{-1}$ nanosheets then enter the solution but will there still associate with positive ions, which depend on which salts are present in the solution. Finally, the nanosheets were re-precipitated by adding $\mathrm{Li}^{+}$ions to the solution in the form of $\mathrm{LiCl}$. Other salts, such as $\mathrm{NaCl}, \mathrm{KCl}$ etc. can be used in this step but here we use Li exclusively. The nanoflakes of $\mathrm{Li}_{x} \mathrm{CoO}_{2}$ had thicknesses, ranging from 30 to $160 \mathrm{~nm}$ as measured by Atomic Force Microscopy (AFM) and Electron Energy Loss Spectroscopy (EELS). Note that the conventional cell of $R \overline{3} m$ has a hexagonal $c$-axis of $\sim 1.4 \mathrm{~nm}$ and contains three $\mathrm{CoO}_{2}$ layers so that the thinnest flakes are still about $60 \mathrm{CoO}_{2}$ layers thick. The composition $\mathrm{Li}_{0.37} \mathrm{CoO}_{2}$ was determined by inductively coupled plasma mass spectroscopy (ICPMS). The $\mathrm{Li}_{0.37} \mathrm{CoO}_{2}$ nanoflakes were deposited in a drop of aqueous chemical solution on top of a degenerately doped $\mathrm{Si}$ wafer with a $300 \mathrm{~nm} \mathrm{SiO}_{2}$ surface layer. There were two batches: one for electronic transport properties, and another one for Transmission Electron Microscopy (TEM) and EELS. For conductivity measurements, individual devices based on $\mathrm{Li}_{0.37} \mathrm{CoO}_{2}$ nanoflakes were obtained by sputtering nickel contacts $(\sim 90 \mathrm{~nm}$ thick $)$ and electron beam lithography. The current-voltage response was measured on $\sim 45-160 \mathrm{~nm}$ nanoflakes, using a Physical Property Measurement System (Quantum Design Inc.). High-temperature annealing at 150, 200, 250, 300, and $350^{\circ} \mathrm{C}$ for $\sim 30$ minutes were performed in vacuum, followed by a gradual cooling $(<10 \mathrm{~K} / \mathrm{min})$.

The ex-situ TEM was performed by transferring annealed nanoflakes onto TEM holey carbon films supported by $\mathrm{Cu}$ grids. The FEG-TEM analysis was performed on a Tecnai F20ST (FEI) operating at $200 \mathrm{kV}$ and equipped with GIF 2000, Gatan, for Energy Filtered TEM (EFTEM) and parallel EELS. The Selected Area Electron Diffraction (SAED), Bright field (BF)/Dark Field (DF) images, energy-filtered HRTEM, and EELS spectra were acquired from individual flakes. The spectra were acquired in diffraction mode, with a camera length of $62 \mathrm{~mm}$ and a GIF entrance aperture of $0.6 \mathrm{~mm}$ leading to collection semi-angle of $2.8 \mathrm{mrad}$. The dispersion was set to $0.1 \mathrm{eV} /$ channel, and a FWHM of the zero loss electrons peak of $\sim 0.6 \mathrm{eV}$ was obtained. The spectra were acquired, as close to the (000) transmitted beam as possible, with the probe radius of $1.15 \mathrm{~nm}-1$ in the horizontal section of reciprocal space, which is represented by a TEM diffraction pattern. This probe radius is $\sim 2-4$ times smaller than the $1 / d_{h k l}$ reciprocal space distances from (000) to neighboring Brillouin zone cen- ters (neighboring diffraction reflections). This allowed to probe electron transitions with $\mathbf{q} \approx 0$ momentum transfer. Essentially one measures the longitudinal $\mathbf{q}=0$ response of the system in this manner, which is theoretically represented by $-\operatorname{Im}\left[\varepsilon^{-1}(\mathbf{q} \approx 0, \omega)\right]$. The spectra were corrected for background and multiple scattering events, and low-loss spectra were deconvoluted by zeroloss (ZL) peak recorded in vacuum, using softwares Digital Micrograph and PEELS (ref P.Fallon, C.A. Walsh, PEELS Program,University of Cambridge, UK, 1996). Fityk was used for fits. The VESTA software ${ }^{24}$ was used for crystal structure visualization.

\section{COMPUTATIONAL METHODS}

The underlying approach for our calculations is density functional theory (DFT) in the generalized gradient approximation (GGA) in the Perdew-Burke-Ernzerhof (PBE) parametrization 25 . Calculations of the band structure and partial densities of states were done using the full-potential linearized muffin-tin-orbital (FPLMTO) method as implemented in questaal.26|27 Convergence parameters for the LMTO calculations were chosen as follows: basis set spdf - spd spherical wave envelope functions plus augmented plane waves with a cut-off of $3 \mathrm{Ry}$, augmentation cutoff $l_{\max }=4, \mathbf{k}$-point mesh, $12 \times 12 \times 12$. We used experimental lattice constants but relaxed the internal structural parameters.

Because some of the materials considered, such as $\mathrm{CoO}$ and $\mathrm{Co}_{3} \mathrm{O}_{4}$ are incorrectly found to be metals within GGA, we also use the DFT+U approach in which onsite Coulomb terms are added for the $\mathrm{Co}-3 d$ orbitals as Hubbard-U parameters. We used the same value of $U=5 \mathrm{eV}$ as in Trimarchiet al. ${ }^{[28} \mathrm{In} \mathrm{LiCoO}_{2}$ in the $R \overline{3} m$ structure, this shifts the already empty $d-e_{g}$ band up relative to the filled bands but keeps the low-spin configuration. In $\mathrm{CoO}$ and $\mathrm{Co}_{3} \mathrm{O}_{4}$ this splits up and down spin orbitals and creates a local magnetic moment. In $\mathrm{Co}_{3} \mathrm{O}_{4}$ we just use the primitive cell and obtain a ferromagnetic solution. In paramagnetic $\mathrm{CoO}$, he situation is more subtle. We use a special quasirandom structure (SQS) approach 28 in a 64 atom cubic supercell, in which up and down spin Co sites are randomly placed. More precisely they are placed such that the pair-correlations between these sites mimic the fully random ones. This is called a polymorphous description of the magnetic moment disorder. Unlike the approach of Trimarchi et al! 28 we here do not displace the atoms in random local fluctuations. Our solution may thus be somewhat different but is similar in spirit. We do not claim here that such fluctuations in position do not occur in response to the random spin directions. Our main goals is to have a qualitatively correct description of the electronic structure with a gap induced by magnetic moment formation.

To describe the $\mathrm{O}-K$ spectra we use the selection rule that for small momentum transfer, transitions from the O- $1 s$ core state to the empty bands are essentially propor- 
tional to the O-p like partial density of states (PDOS). One should note, however, that for high energy states, this does not necessarily correspond to the atomic O- $2 p$ states but rather higher excited atomic states or merely the tails of surrounding atom wave functions expanded in spherical harmonics inside the O-sphere. It is well known that the O-1s core hole presence may modify the higher state O- $p$ like PDOS and hence we include the core-hole explicitly in the calculation. This is known as the final state rule in X-ray absorption spectroscopy but the same applies in EELS. Core holes are created in a randomly chosen oxygen site in a supercell, after which the total electron density is iterated to self consistency. Using this new self-consistent wave function, the spectrum is calculated including the effect of the momentum or dipole matrix elements,

$$
S(\epsilon) \propto \sum_{n \mathbf{k}}\left|\left\langle\psi_{c}|\mathbf{p}| \psi_{n \mathbf{k}}\right\rangle\right|^{2}\left(1-f_{n \mathbf{k}}\right) \delta\left(\epsilon-\epsilon_{n \mathbf{k}}+\epsilon_{c}\right),
$$

with $\epsilon_{n \mathbf{k}}, \psi_{n \mathbf{k}}$ the band Kohn-Sham eigenvalue and the corresponding wavefunction, $\epsilon_{c}, \psi_{c}$ the core energy and wave function, and $f_{n \mathbf{k}}$ the Fermi-Dirac occupation factor. Because we do not attempt to calculate the absolute core-level spectrum edge, the $\epsilon_{c}$ is not actually calculated and set to zero and the first peak is aligned with the experimental spectra. For the $R \overline{3} m$ structure a $3 \times 3 \times 3$ supercell is used. Similar size supercells are used in the other cases. A Gaussian broadening of $\sim 1 \mathrm{eV}$ is applied to these spectra to represent instrumental broadening for easier comparison with the experiment. We have checked that including the core-hole is not crucial to represent the shape of the spectra and even a simple $p$-PDOS gives actually similar results. Thus we use the latter for the SQS representing the rocksalt paramagnetic $\mathrm{CoO}$ for which it was difficult to find a converged solution using the FPLMTO code. Instead we used the GPAW code with an LCAO basis set which is also used for the EELS, as described next.

The measured low energy EELS are compared with calculations of the loss-function $-\operatorname{Im}\left[\varepsilon^{-1}(\mathbf{q}, \omega)\right.$ at small finite q. These calculations are performed within the generalized random phase approximation (RPA) using GPAW 31 which uses the projector augmented wave (PAW) method 34 . The kinetic energy cut-off for the plane wave basis set is taken to be $700 \mathrm{eV}$ with a $\mathbf{k}$-point grid of $20 \times 20 \times 20$. The band structures obtained with this method were checked to be in excellent agreement with the all-electron FP-LMTO results.

To calculate the loss function, we start from the noninteracting charge-charge response function $\left(\chi_{\mathbf{G G}^{\prime}}^{0}(\mathbf{q}, \omega)\right)$ obtained from the Adler-Wiser formula 35136

$$
\begin{aligned}
\chi_{\mathbf{G G}^{\prime}}^{0}(\mathbf{q}, \omega)= & \frac{1}{\Omega} \sum_{\mathbf{k}} \sum_{n, n^{\prime}} \frac{f_{n \mathbf{k}}-f_{n^{\prime} \mathbf{k}+\mathbf{q}}}{\omega+\epsilon_{n \mathbf{k}}-\epsilon_{n^{\prime} \mathbf{k}+\mathbf{q}}+i \eta} \\
& \left\langle\psi_{n \mathbf{k}}\left|e^{-i(\mathbf{q}+\mathbf{G}) \cdot \mathbf{r}}\right| \psi_{n^{\prime} \mathbf{k}+\mathbf{q}}\right\rangle_{\Omega_{\text {cell }}} \\
& \left\langle\psi_{n \mathbf{k}}\left|e^{i\left(\mathbf{q}+\mathbf{G}^{\prime}\right) \cdot \mathbf{r}^{\prime}}\right| \psi_{n^{\prime} \mathbf{k}+\mathbf{q}}\right\rangle_{\Omega_{\text {cell }}},
\end{aligned}
$$

where $\Omega$ is the crystal volume. Here, $\mathbf{G}$ and $\mathbf{q}$ are the reciprocal lattice vector and the wave vector of the charge density perturbation respectively.

Within Time Dependent Density Functional Theory (TDDFT), one can write the interacting charge-charge response function by solving a Dyson equation of the form

$$
\begin{aligned}
\chi_{\mathbf{G G}^{\prime}}(\mathbf{q}, \omega)= & \chi_{\mathbf{G G}^{\prime}}^{0}(\mathbf{q}, \omega)+ \\
& \sum_{\mathbf{G}_{1} \mathbf{G}_{2}} \chi_{\mathbf{G G}_{1}}^{0}(\mathbf{q}, \omega) K_{\mathbf{G}_{1} \mathbf{G}_{2}}(\mathbf{q}) \chi_{\mathbf{G}_{2} \mathbf{G}^{\prime}}(\mathbf{q}, \omega) .
\end{aligned}
$$

Here the kernel $K_{\mathbf{G}_{1} \mathbf{G}_{2}}$ is treated in the random phase approximation (RPA) and hence only includes the Coulomb part.

$$
K_{\mathbf{G}_{1} \mathbf{G}_{2}}(\mathbf{q})=\frac{4 \pi}{\left|\mathbf{q}+\mathbf{G}_{1}\right|^{2}} \delta_{\mathbf{G}_{1} \mathbf{G}_{2}}
$$

Using $\chi_{\mathbf{G G}^{\prime}}(\mathbf{q}, \omega)$, the macroscopic dielectric function $\epsilon_{M}$ is obtained to be

$$
\varepsilon_{M}^{-1}(\mathbf{q}, \omega)=1+\frac{4 \pi}{|\mathbf{q}+\mathbf{G}|^{2}} \chi_{\mathbf{G}=\mathbf{0}, \mathbf{G}^{\prime}=\mathbf{0}}(\mathbf{q}, \omega)
$$

The $\mathbf{q}$ dependent dynamical loss function can be directly related to the loss function which is calculated as

$$
\mathcal{A}_{\mathrm{EELS}}(\mathbf{q}, \omega)=-\operatorname{Im}\left[\varepsilon_{M}^{-1}(\mathbf{q}, \omega)\right]
$$

In addition, we may neglect local field effects (LFE), in which case

$$
\varepsilon_{\mathbf{G}, \mathbf{G}^{\prime}}^{N L F}(\mathbf{q}, \omega)=\delta_{\mathbf{G}, \mathbf{G}^{\prime}}-\frac{4 \pi}{|\mathbf{q}+\mathbf{G}|^{2}} \chi_{\mathbf{G}, \mathbf{G}^{\prime}}^{0}(\mathbf{q}, \omega)
$$

and

$$
\left.\varepsilon_{M}^{-1}(\mathbf{q}, \omega) \approx 1 / \varepsilon_{\mathbf{G}=\mathbf{0}, \mathbf{G}^{\prime}=\mathbf{0}}^{N L F E}(\mathbf{q}, \omega)\right]
$$

\section{RESULTS}

\section{A. TEM diffraction observations}

Fig. 11 illustrates the structure evolution of the $\mathrm{Li}_{x} \mathrm{CoO}_{2}$ nanoflakes with temperature, determined through the changes of TEM SAED patterns. The nanosheets typically were found lying with their octahedral sheets parallel to the carbon film. Before annealing, the diffraction pattern of a flake (Fig. 1b) showed two arrays of spots, an intense one circled in yellow that corresponds to a single crystal with $R \overline{3} \mathrm{~m}$ symmetry observed in the [0001] zone axis, and a fainter array of spots circled in red, that corresponds to the formation of coherent nano-domains with the $C 2 / m$ symmetry. The latter is due to the slight distortion of the cell due to the loss of lithium (Fig. 1a) and the resulting repulsion between the $\mathrm{CoO}_{2}$ layers. This structure corresponds to a random distribution of vacancies on the $\mathrm{Li}$ sites. It was shown before ${ }^{37 \mid 38}$ that the "O3"-type $C 2 / m$ phase (Fig. 

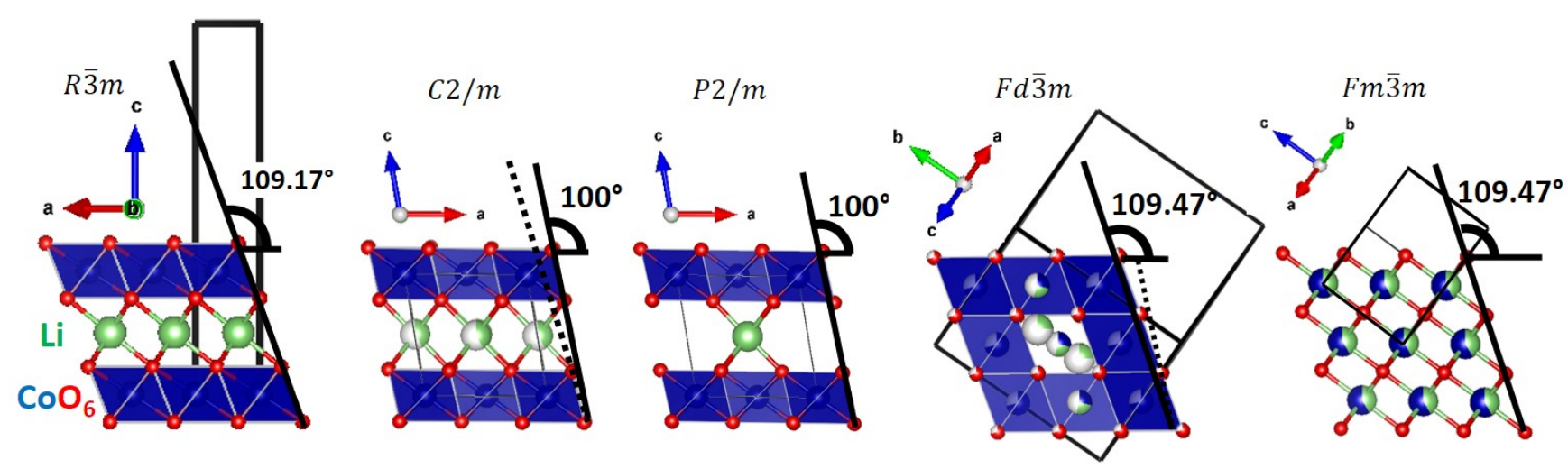

(a) Schematic crystal structures.

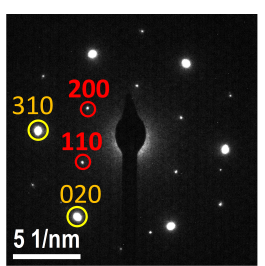

(b) $20^{\circ} \mathrm{C}$ $R \overline{3} m+C 2 / m$

$\langle 001\rangle$.

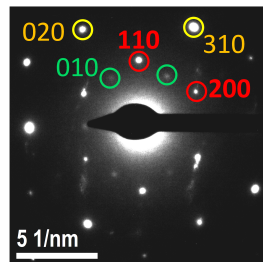

(c) $150^{\circ} \mathrm{C}$

$R \overline{3} m+C 2 / m$

$\langle 001\rangle$.

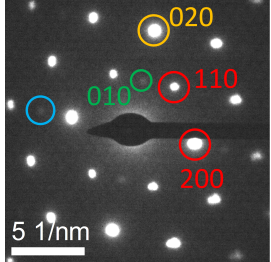

(d) $200^{\circ} \mathrm{C}$

$R \overline{3} m+C 2 / m+P 2 / m$

$\langle 001\rangle$.

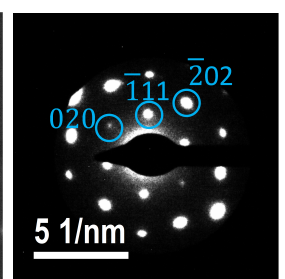

(e) $250^{\circ} \mathrm{C}$

$F d \overline{3} m$

$\langle 101\rangle$.

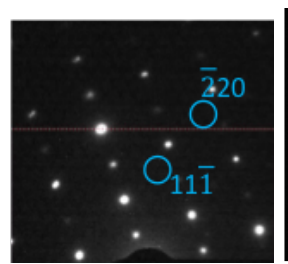

(f) $250^{\circ} \mathrm{C}$ $F d \overline{3} m$ $\langle 112\rangle$.

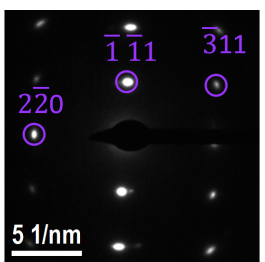

(g) $350^{\circ} \mathrm{C}$

$F m \overline{3} m$

$\langle 112\rangle$.

Fig. 1: Structure evolution upon annealing: (a) the schematic shows a repeatable part of structure which is preserved on phase transformations (not a unit cell). The pure $\mathrm{CoO}_{6}$ atomic columns consist of blue octahedra.

(b-g)SAED patterns for different annealing temperatures.

1a) was stabilized for $x \approx 0.2-0.5$. A unique crystal orientation relationship with the $R \overline{3} m$ matrix was found,

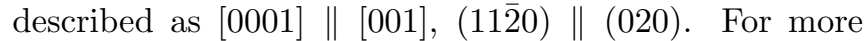
structural details, see Supplementary Table ST1 39

After annealing at $150^{\circ} \mathrm{C}$, the monoclinic $\mathrm{C} 2 / \mathrm{m}$ domains were coarsening reaching the size of the former $R \overline{3} m$ domains (Supplementary Fig. S2), ${ }^{39}$ as also evidenced in Fig. 1. through growing intensity of the corresponding reflections (in red). The prolonged TEM observation favored the appearance of additional 010 spots (in green), forbidden by the $\mathrm{C}$ lattice symmetry. These additional spots evidence the beginning of the phase transformation into the $P 2 / m$ phase on beam damage. In the $P 2 / m$ phase, $1: 1$ in-plane ordering of the Li-vacancy in $\mathrm{Li}_{0.5} \mathrm{CoO}_{2}$ takes place (Fig. 1a), as compared to the $C 2 / m$ phase. In Fig. $1 \mathrm{c}$, the three pairs of new reflections are attributed to three domain orientations 41 , which we also observed through energy filtered HRTEM (Supplementary Fig. S1(2)) ${ }^{[39}$ For $T=200^{\circ} \mathrm{C}$ (Fig. 1d), the $C 2 / m$ and $P 2 / m$ phases coexist. The phase transformation of monoclinic phase into a cubic spineltype $F d \overline{3} m$ forming nano-domains was seen in HRTEM (Supplementary figs. S1 $(3,4) \cdot \frac{39}{39}$ The orientation relationship (Figs. 1 $1 \mathrm{~d}-\mathrm{e}$ ) was found to be $[010]_{P} \|[101]_{F}$ and $[001]_{P} \| \approx[111]_{F}$.

The phase transformation to spinel was confirmed after annealing at $250^{\circ} \mathrm{C}$ (Fig. 1 1 d-e, where the $F d \overline{3} m$ phase was macroscopic (Fig. 11d-e and Supplementary
Fig.S1) $[39$ The transformation results from inter-layer diffusion of $\mathrm{Co} / \mathrm{Li}$, with octahedral and tetrahedral site occupancies. Combining our SAED with characteristic spot intensities (Fig. 1e), the EFTEM rod-like contrast (Supplementary Fig. S1(4)),,$[9$ and simulations of SAED/EFTEM of known spinel phases ${ }^{42 / 43}$, we proposed the structure shown in Fig. 1a, with 8a tetragonal sites partially occupied by $\mathrm{Li}$ and $16 \mathrm{~d}$ (blue octahedra) and $16 \mathrm{c}$ sites alternating in the non-diagonal columns occupied by Co. The simulation of diffraction patterns were made in the $\langle 211\rangle$ zone axis, since it allowed a better discrimination of the spinel types by the relative intensities of their diffraction spots than in the $\langle 111\rangle$ zone axis (Supplementary Fig. S3a) ${ }^{39}$ The EFTEM contrast was simulated in [101] zone axis (Supplementary Fig. S1(4-5))! 39 We propose the suitable atomic site occupations, which are given in Supplementary table ST1.39 More studies are necessary to determine the precise site occupation. For $T=300^{\circ} \mathrm{C}$, the flakes stayed in the $F d \overline{3} m$ phase (Supplementary figs. S1-5 and S2-b) $\underline{39}$

For $T=350^{\circ} \mathrm{C}$, some of the flakes transformed into the rocksalt $F m \overline{3} m$ phase (Fig, 1a) with about half lattice parameter $(4.2 \AA)$ and the same orientation of the $a$, $b, c$ lattice vectors. The transition is due to full mixing of $\mathrm{Co}$ and $\mathrm{Li}$ in $4 \mathrm{a}$ octahedral sites of $F m \overline{3} m$ (fig. 1a) corresponding to the former $16 \mathrm{c}$ and $16 \mathrm{~d}$ sites of the $F d \overline{3} \mathrm{~m}$ structure. The partial phase transition is explained by different flake thicknesses, since it controls the rate of 
(a)

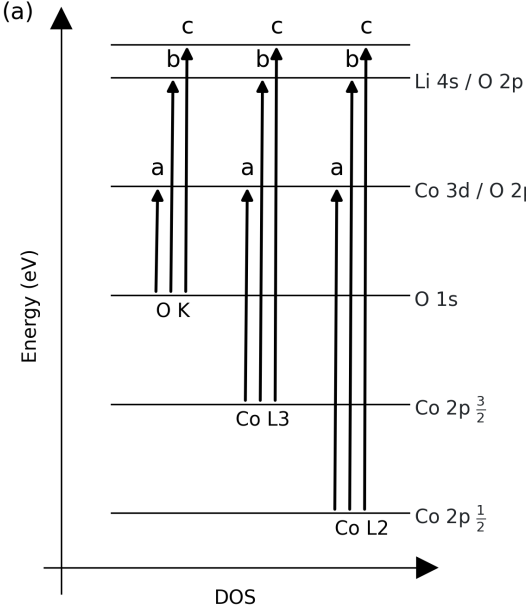

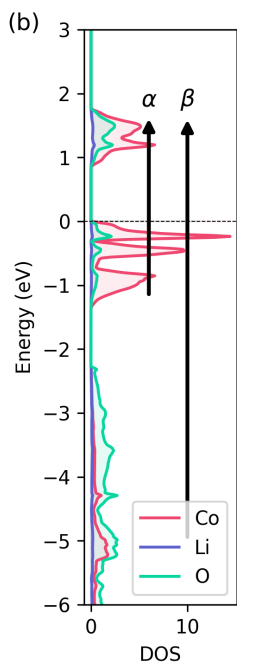

Fig. 2: Schematic of energy transitions.

oxygen loss 44 . On top of newly formed $F m \overline{3} m$, a remnant $F d \overline{3} m$ phase was observed, which was similar to a fully disordered spine ${ }^{42}$. The energy filtered HRTEM (Supplementary fig.S1-6) ${ }^{39}$ showed the contrast, typical for spinel-like structure with the $8.3 \AA$ lattice parameter, but with empty 8a sites (Supplementary fig. S3c).

\section{B. Overview of electronic transitions}

Electronic structure changes induced by the structural modifications are studied in the following parts through EELS spectra of O- $K$ and Co-L edges. Fluctuations near the absorption edges are related to the probability of electron transitions from occupied to unoccupied electron levels. A schematic overview of the electronic transitions discussed here is given in Fig2

The GGA band structure and DOS of $\mathrm{LiCoO}_{2}$ in the $R \overline{3} m$ structure are shown in Fig. 3 Please note the different PDOS scale in the higher lying conduction band region. The top of the valence band is Co- $d-t_{2 g}$ like, while deeper bands have more O- $2 p$ character. The lowest conduction band is dominated by Co- $3 d$ while the higher bands in the range $6-10 \mathrm{eV}$ have mainly $\mathrm{Li}-2 s 2 p$ and O-antibonding contributions. There is also a Co$4 s$ contribution which however is stronger between 12-14 $\mathrm{eV}$. The bands in this energy range have strong dispersion and are essentially free-electron like. In the GGA, the band gap is somewhat underestimated and amounts to $0.89 \mathrm{eV}$ for the indirect gap.

\section{O- $K$ edge spectra}

In Fig. 4, the inset shows the typical EELS O- $K$ edge spectrum for $20^{\circ} \mathrm{C}$ (non-annealed) sample (spectra recorded at all studied temperatures on several particles
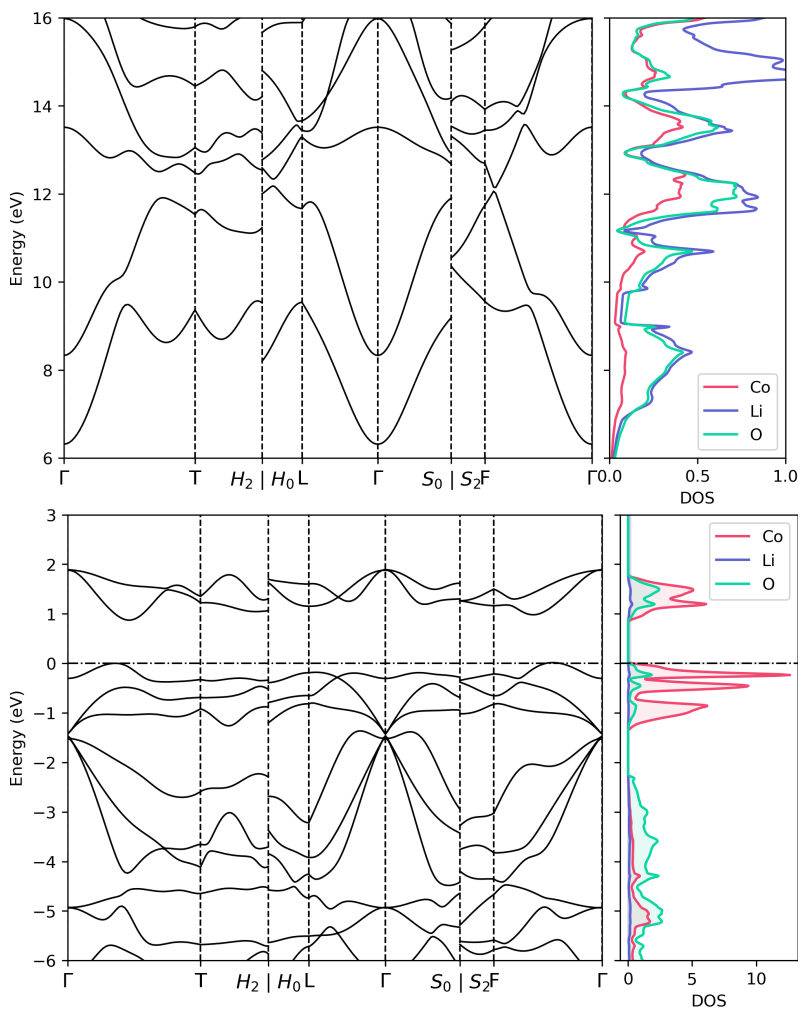

Fig. 3: GGA band structure and $\mathrm{PDOS}$ of $\mathrm{LiCoO}_{2}$ in $R \overline{3} m$ structure in two energy intervals.

and processed to build this figure are given in Supplementary fig. S4) $\frac{39}{39}$ The pre-peak "a" (Fig. 20 comes from electron transitions from the $\mathrm{O}-1 s$ core level towards the lowest conduction band, ${ }^{455}$ which is the Co-3d$e_{g}$ band, where $e_{g}$ refers to the irreducible representation in the octahedral group of the Co octahedral environment. These are antibonding hybridized Co- $3 d-\mathrm{O}-2 p$ states. The components "b" and "c" are due to transitions towards higher conduction bands, which involve both the Li- $2 s$ and $\mathrm{Co}-4 s$ derived bands antibondng with O- $2 p$ and are essentially free-electron like bands.

According to Zhao et al ${ }^{46}$ the relative intensity of the pre-peak "a" to the " $\mathrm{b} / \mathrm{c}$ " peaks is connected to the degree of Co $3 d-\mathrm{O} 2 p$ hybridization. We thus studied this ratio of peak intensities as function of annealing temperature, $T$, as shown in Fig 4 . For annealed nanoflakes, in the range $T=20^{\circ} \mathrm{C}-200^{\circ} \mathrm{C}$, the ratio first increased, which would indicate an increase of the Co- $3 d-\mathrm{O}-2 p$ hybridization for the $R \overline{3} m \rightarrow C 2 / m \rightarrow P 2 / m$ phase sequence. On the other hand, for $T=200^{\circ} \mathrm{C}$ to $350^{\circ} \mathrm{C}$ and the transition $F d \overline{3} m \rightarrow F m \overline{3} m$, the $A(a) / A(b c)$ ratio decreased dramatically.

Alternatively, we compare these spectra directly with calculated $\mathrm{O}-K$ spectra for various model systems in Fig. 5. First, in (a1) we compare different computational approaches for $\mathrm{LiCoO}_{2}$ in the $R \overline{3} m$ structure. This shows that the calculation taking the core-hole and O- $1 s$ to band states matrix elements explicitly into account ac- 


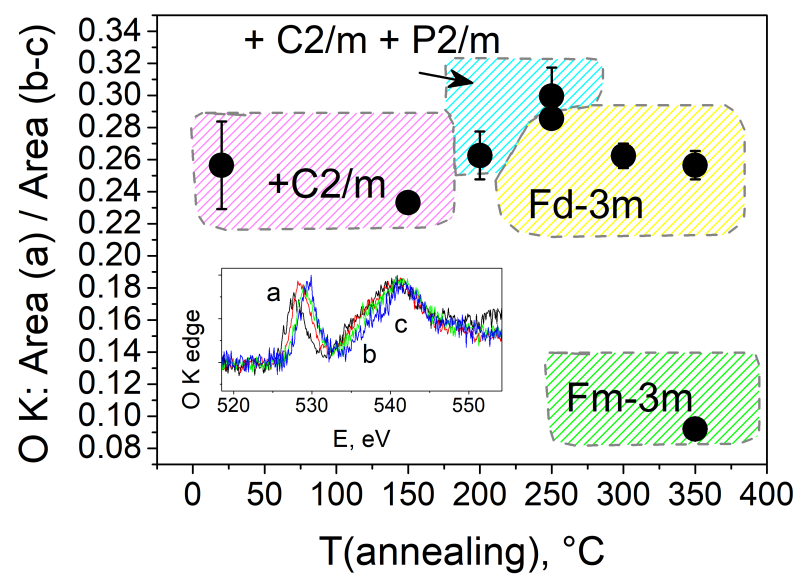

Fig. 4: EELS study of O K edge: (a) the ratio of integrated area $A(a) / A(b, c)$ of the pre-peak "a" versus main peak components "b" and "c", as identified in the inset, which shows the spectrum of $\mathrm{Li}_{0.37} \mathrm{CoO}_{2}$ at $20^{\circ} \mathrm{C}$.

cording to Eq. 1 is almost indistinguishable from the simple O- $2 p$ PDOS. Adding $U$ to the GGA just shifts the Co- $d$ bands up slightly but the spectra in either of these models agree well with the experimental spectrum in (b1). The zero of energy in our calculation is the highest occupied band (VBM) but was then shifted by $527 \mathrm{eV}$ to align the first "a" peak with experiment. A Gaussian broadening by $\sim 1 \mathrm{eV}$ is applied to our calculated spectrum after removing the filled part of the PDOS. The unbroadened spectrum is shown in thinner lines underneath the shading. We expect negligible changes in the closely related $C 2 / m$ and $P 2 / m$ phase. In the experimental panels (b1-b6) the black thicker line is a smoothed average over different samples. The sample details are provided in Supplemental Information Fig. S4(a) $\underline{39}$

Next, in panels (a2-a4) we show GGA+U results for the O- $p$ PDOS for various models in an attempt to qualitatively understand changes in these spectra. For the $F d \overline{3} m$ calculations, we used a model provided by Materials Project ${ }^{48}$ with the above spacegroup. It contains both $\mathrm{Li}$ and Co exclusively in octahedral sites. One can see that there is little qualitative change between the $R \overline{3} m$ and $F d \overline{3} m$ spectra, just as there is little change between the $P 2 / m$ and $F d \overline{3} m$ spectrum in panel (b4). We also calculated a $F m \overline{3} m$ spectrum using a supercell of the rocksalt structure with a random occupation of $\mathrm{Li}$ and $\mathrm{Co}$ atoms in a ratio of $3 / 7 \mathrm{Li} / \mathrm{Co}$. Finally, we also considered spinel $\mathrm{Co}_{3} \mathrm{O}_{4}$ and rocksalt $\mathrm{CoO}$ without any Li.

We note that in the rocksalt phase in GGA the $t_{2 g}$ to $e_{g}$ crystal field splitting in the octahedral environment is reduced compared to that in $R \overline{3} m$ because of the larger Co-O distance. On the other hand, the bands have significantly larger band dispersions, related to the more $3 \mathrm{D}$ network type arrangement of $\mathrm{Co}$ and $\mathrm{Li}$ compared to the layered structure in $R \overline{3} \mathrm{~m}$. Because of this we no
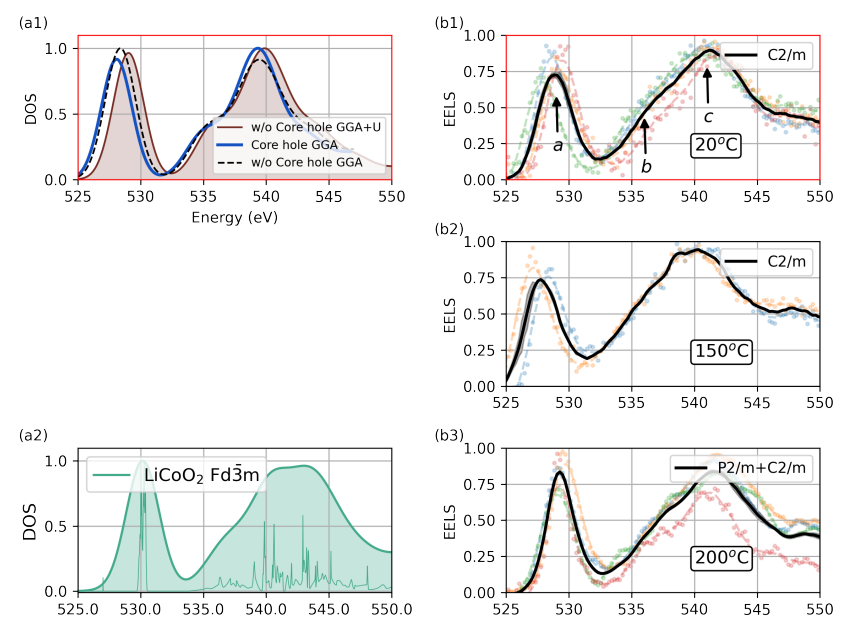

(a3)
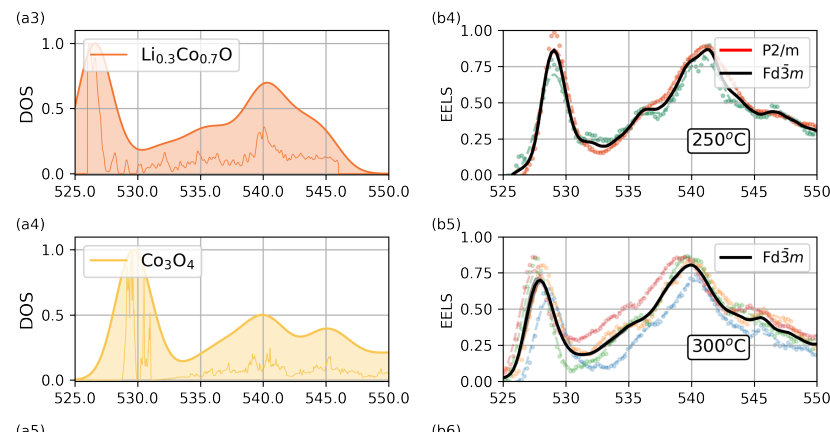

(a5)
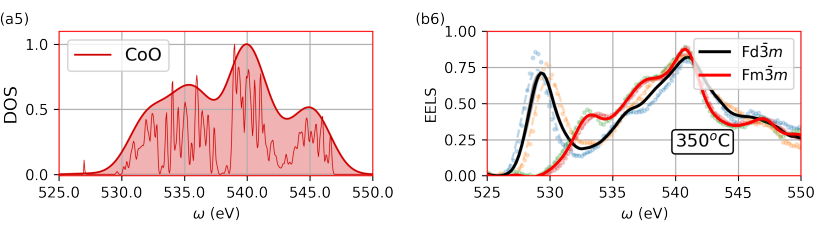

Fig. 5: (a) Simulated O- $K$ spectra for various systems: (a1) comparison of Eq. (1) including O-1s core hole and matrix elements explicitly with simple O- $p$ PDOS both in GGA and with O- $p$ PDOS in GGA $+\mathrm{U}(U=5 \mathrm{eV})$ for $\mathrm{LiCoO}_{2}$ in $R \overline{3} m$ structure, (a2-a5) O- $p$ PDOS in

GGA+U for: (a2) $\mathrm{LiCoO}_{2}$ in $F d \overline{3} m$ model, (a3) $\mathrm{Li}_{0.3} \mathrm{Co}_{0.7} \mathrm{O}$ in $F m \overline{3} m$ structure, (a4) $\mathrm{Co}_{3} \mathrm{O}_{4}$ in spinel

$F d \overline{3} m$ structure and (a5) $\mathrm{CoO}$ in rocksalt $F m \overline{3} m$ structure. (b) Experimental EELS for different samples (shown in scatter plots), with average data smoothened using Savitzky-Golay filter $\frac{[47}{47}$ shown in red/black for various temperatures

longer have a gap between separated $t_{2 g}$ and $e_{g}$ bands but these two overlap and lead to a metallic band structure, in disagreement with experiment. This is why we need to use GGA+U to obtain a qualitatively meaningful electronic structure. The gap in such systems arises from the formation of magnetic moments and correlation effects in the partially filled Co- $3 d$ derived bands. For $\mathrm{Co}_{3} \mathrm{O}_{4}$ we obtain a ferromagnetic band structure in the primitive cell when using GGA+U. For $\mathrm{CoO}$ we use an SQS model with random distribution of up and down spins to model the paramagnetic state. Likewise for the 
Li containing $F m \overline{3} m$ structure we use the SQS approach to model both the $\mathrm{Li} / \mathrm{Co}$ random location and up and down spins on Co sites. Band structures for these cases are shown in Supplemental Information Figs. S7-S9 39 Even with GGA+U, for the $\mathrm{Li}_{0.3} \mathrm{Co}_{0.7} \mathrm{O} F m \overline{3} m$ structure we obtain a metallic band structure with the Fermi level close to the top of the $d t_{2 g}$ band (Fig. S9).

We can see that qualitatively all the simulated $\mathrm{O}-K$ spectra stay rather similar with similar "a" and "b/c" peak relative positions and intensity ratio. The exception is $\mathrm{CoO}$ where the a peak is signficantly less intense and closer to the $\mathrm{b} / \mathrm{c}$ peaks and this agrees with the experimental observation for the $F m \overline{3} m$ spectra. From the entire series of experimental data, taking in to account the sample to sample variation, the most obvious change occurs for the $F m \overline{3} m$ phase at $350^{\circ} \mathrm{C}$. From Fig. S4(a) and Fig 5 (b6), one can see that the strong drop in $A(a) / A(b c)$ is related to a shift in the "a" peak to higher energies in qualitative agreement with the $\mathrm{CoO}$ result in Fig 5(a5). This can also be seen in the sim-

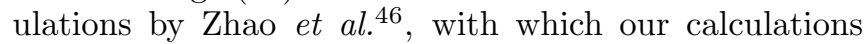
agree quite well, even though their calculation did not include the core-hole induced changes in the density of states and was purely GGA. The experimental spectra for the $F m \overline{3} m$ phase are much more resemblant of the pure $\mathrm{CoO}$ phase than of the $F m \overline{3} m \mathrm{Li}_{0.3} \mathrm{Co}_{0.7} \mathrm{O}$ model we calculated. This is an indication that at these higher temperatures, the system may have lost some Li from the rocksalt phase parts of the sample by diffusion into other regions.

\section{Co- $L_{2,3}$ edge spectra}

The origin of the Co $L_{3}, L_{2}$ edge components is explained schematically in Fig. 22. They correpond to transitions from core levels Co $2 p_{3 / 2}\left(L_{3}\right)$ and Co $2 p_{1 / 2}$ $\left(L_{2}\right)$. Both $L_{3}$ and $L_{2}$ include three components each, marked as "a-c" and "d-f". Importantly, the intensity ratio $A\left(L_{3}\right) / A\left(L_{2}\right)$ in $3 d$ transition metals is recognized as a parameter which reflects the cation valence $e^{46 / 49 \mid 50}$. This is related to many body effects in these open shell states. Fig. 6 presents the variation of Co valence, deduced from the calibration curve for $A\left(L_{3}\right) / A\left(L_{2}\right)$, presented in Supplementary Fig. S5 $\stackrel{39}{ }$ The step-like changes of the Co valence in Fig 6 are connected with the phase changes. Prior to annealing the mean $\mathrm{Co}$ valence of $\mathrm{Li}_{0.37} \mathrm{CoO}_{2}$ was around 3.3. A charge compensation of the Li loss handled by Co only would give a valence of 3.63. The lower experimental valence, together with the Co $3 d-\mathrm{O}$ $2 p$ hybridization revealed by the O- $K$ pre-peak, indicates a contribution from $\mathrm{O}-2 p$ holes in the charge compensation. This is also consistent with findings by Wolverton and Zunger ${ }^{51}$ that within a sphere around Co, little change in charge density is found upon Li loss but rather a change in Co-O bonding. At $150^{\circ} \mathrm{C}$, the $\mathrm{Co}$ valence is lowered to 3.15 , probably due to the increase of covalency and $\mathrm{Co}-\mathrm{O}$ hybridizations in the growing $\mathrm{C} 2 / \mathrm{m}$

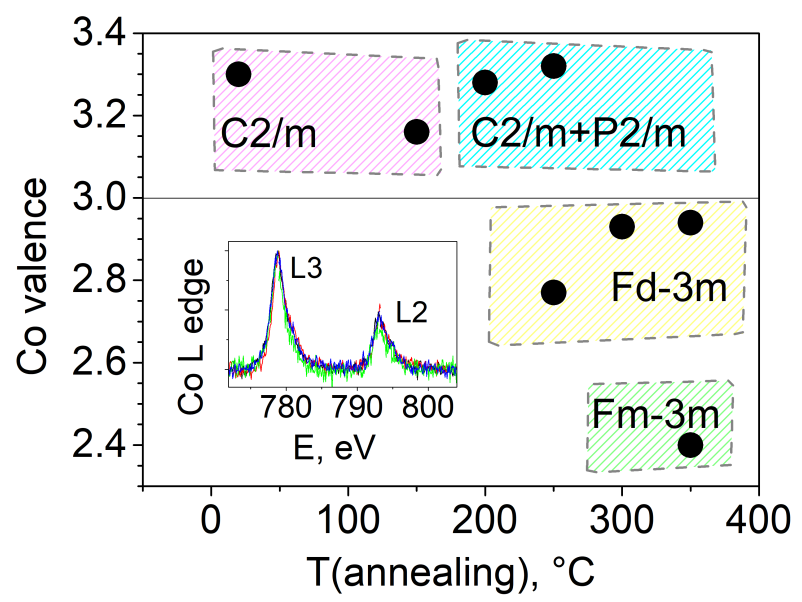

Fig. 6: Average Co valence as deduced from the $\mathrm{L}_{3} / \mathrm{L}_{2} 2$ edge peak ratio.

phase. The gradual growth of $P 2 / m$ domains induces an increase of the mean Co valence. The $1: 1$ ordering of the $\mathrm{Li}$ vacancies drives the charge ordering on $\mathrm{Co}$ sites, inducing Co atoms which donate to $\mathrm{O}$ more electronic charge than in a random vacancy distribution. $\frac{18] 40}{}$ Essentially, $\mathrm{Co}^{4+}$ is formed near the $\mathrm{Li}$ vacancies. This is, probably, the reason why the Co valence is larger in $P 2 / m$ phase than in $C 2 / m$, although still with a degree of covalency in the Co-O bond. Finally, for $T>250^{\circ} \mathrm{C}$, $F d \overline{3} m$ and $F m \overline{3} m$ phases have reduced average Co valence $<3+$, which evidences the increasing presence of reduced cations $\mathrm{Co}^{2+}$. The pure monoxide $\mathrm{CoO}$ has also the $F m \overline{3} m$ rocksalt structure ${ }^{[52}$ The reduction in average valence by the presence of $\mathrm{Co}^{2+}$ in the rocskalt phase is consistent with a loss of $\mathrm{Li}$ from these regions found in the previous section from the $\mathrm{O}-K$ spectra.

\section{E. Low energy EELS}

Fig. 7 shows the measured low energy EELS for two temperatures. The spectrum is deconvolved into an asymmetric $\alpha$ peak at about $3 \mathrm{eV}$ and rather broad $\beta$ peak at about $7.5 \mathrm{eV}$ and a weak higher feature labeled $\beta^{\prime \prime}$. As indicated in Fig. 2 one may loosely associate the $\alpha$ peak to transitions from the topmost Co- $t_{2 g}$ part of the valence band to the $e_{g}$ conduction bands and the $\beta$ peak to transitions from, a peak in density of states deeper in the valence band with more $\mathrm{O}-2 p$ character to the same conduction band. In between there is a region of low density of valence band states which explains qualitatively why the $\alpha$-peak has a low density tail toward higher energies and asymmetric shape. As shown in Supplementary Information (Fig. S10), ${ }^{39}$ a simple convolution of the occupied and empty densities of states, ignoring any momentum conservation provides this type of general shape with an asymmetric $\alpha$ and a broad $\beta$ peak.

However, a better approach is to compare these EELS 


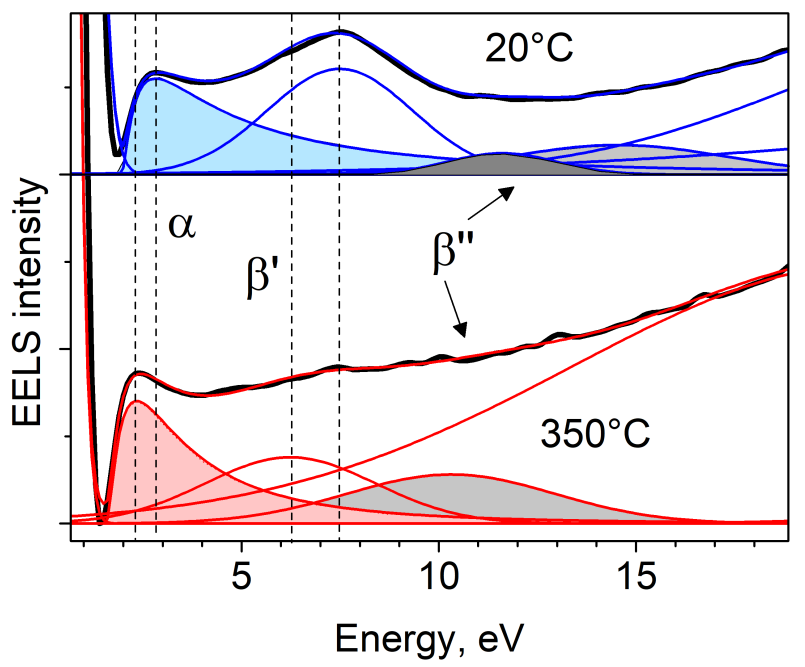

(a)

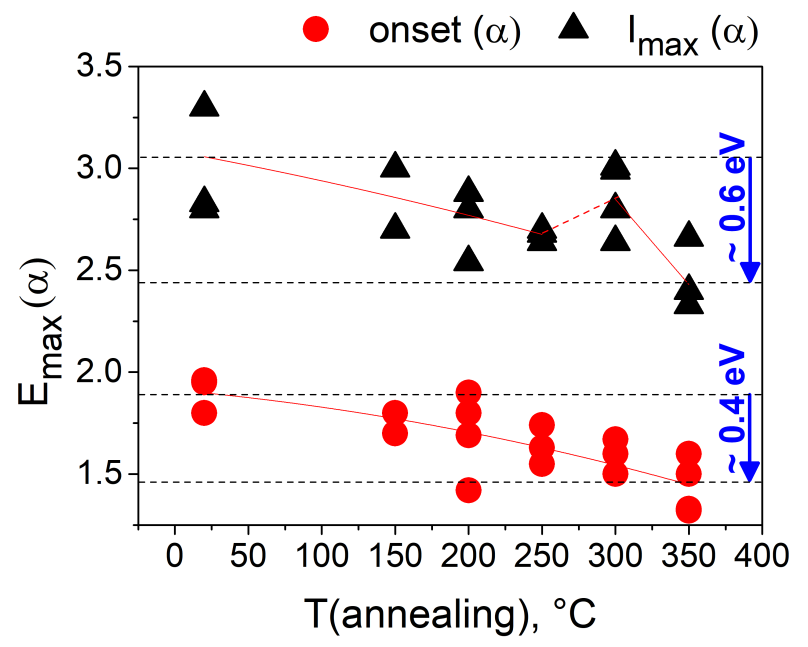

(b)

Fig. 7: Low energy loss spectra for near-bandgap electronic structure: (a) the fit model shown for two spectra after annealing at $20^{\circ} \mathrm{C}$ and $350^{\circ} \mathrm{C}$, (b) onset of transition $\alpha$ and it's maximum plotted for all nanoflakes.

spectra with the calculated loss spectrum, which is shown in Fig. 8. As mentioned in the computational methods section, this corresponds to the $q \approx 0$ energy longitudinal response function. To be clear this corresponds to vertical transitions but integrated over the whole Brillouin zone. We here show calculations as function $\mathbf{q}$ and both including and neglecting local field effects. The first weak peak around $2.5 \mathrm{eV}$ agrees well with the experimental $\alpha$ peak. The next strong peak centered at $\sim 8 \mathrm{eV}$ agrees well with the experimental $\beta$-peak. Even finer structure be recognized in the experiment as shoulder structures. The broad strong peak between $20-30 \mathrm{eV}$ corresponds to the plasmon. Using, $\omega_{P}=\sqrt{4 \pi n e^{2} / m_{e}}$ including 18 valence electrons, so not including the O-2 $s$ electrons as
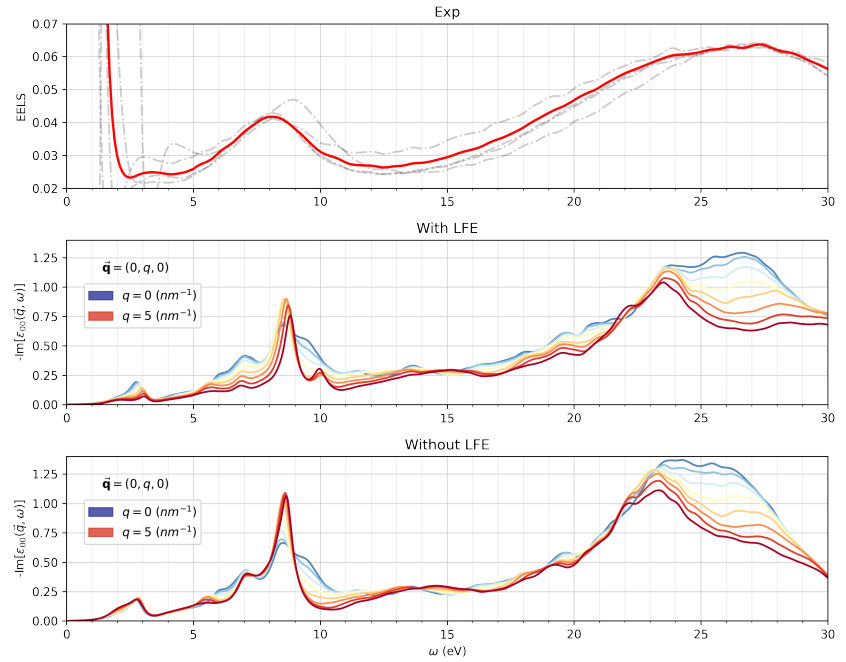

Fig. 8: EELS, from to to bottom: (a) experimental data at $20^{\circ} \mathrm{C}$ the red curve is an average over three samples; Calculated $\mathcal{A}_{\mathrm{ELSS}}(\mathbf{q}, \omega)$ at $\mathbf{q}=(0, q, 0)$ for $q=0$ (blue)

to $q=0.5$ (red) with (b) and without(c) Local Field effects.

valence, one obtains a plasmon energy of $27 \mathrm{eV}$, while including the O- $2 s$ would give $30 \mathrm{eV}$. On the other hand the $\mathrm{O}-2 s$ derived bands lie about $20 \mathrm{eV}$ below the VBM, so band-to-band transitions from these to the $e_{g}$ conduction band are also expected in this energy range, overlapping with the dominant plasmon peak.

In this figure we show the spectrum as function $\mathbf{q}$ up to about $5 \mathrm{~nm}^{-1}$. One may see that including local field effects, the $\alpha$ peak gradually becomes smaller as $\mathbf{q}$ increases but this effect is not seen when local field effects are neglected. In the present experiment, we estimate that we integrate the spectrum near $\mathbf{q}=0$ up to about $q=5 \mathrm{~nm}^{-1}$. To measure the spectrum as function of $\mathbf{q}$ one would need to vary the central $\mathbf{q}$ away from the (000) spot in small steps and within an even smaller range or spot size $\delta q$. Such measurements were done for the Li-1s loss spectrum in Ref. 53.

Fig. 7 shows that both the onset and the location of the maximum intensity of the $\alpha$-peak shift to the lower energy on increase of the annealing temperature. Within the error of measurements, we observe a decrease of these values by $\sim 0.4$ and $\sim 0.6 \mathrm{eV}$ respectively. This decrease indicates a decrease of the $t_{2 g}-e_{g}$ gap and it agrees with the prediction 5455 of a band gap decrease with Co valence decrease. The decrease in valence is also correlated with changes in Co- $3 d-\mathrm{O}-2 p$ hybridization or degree of covalency as indicated by the $\mathrm{O}-\mathrm{K}$ spectra.

On the other hand, in $\mathrm{CoO}$ and $\mathrm{Co}_{3} \mathrm{O}_{4}$ the correlated electronic structure can no longer be explained purely within a standard DFT band-structure picture, though it is still possible to obtain a gap within DFT $+\mathrm{U}$ even for the disordered paramagnetic phase using a polymorphous description, which includes local symmetry 
breaking and spatial fluctuations ${ }^{28}$ Trimarchi et al. ${ }^{28}$ obtain a gap of $2.25 \mathrm{eV}$ for $\mathrm{CoO}$ in the paramagnetic rocksalt structure. Our own calculations of the band structure within GGA+U (given in the Supplemental Information $\mathrm{S} 8)^{\sqrt{39}}$ give a gap of 1.94 in paramagnetic $\mathrm{CoO}$ and this is indeed somewhat smaller than the gap obtained for $\mathrm{LiCoO}_{2}$ in either the $R \overline{3} m(2.746 \mathrm{eV})$ or $F d \overline{3} m$ phases $(2.710 \mathrm{eV})$.

From previous literature, the prediction of decreasing gaps $\frac{54 / 55}{}$ is found to hold for $\mathrm{Li}_{x} \mathrm{CoO}_{2}$ with varying $x$. In the extreme case of $x=0$, pure $\mathrm{Co}_{3} \mathrm{O}_{4}$ in the $F d \overline{3} m$ phase also has been reported to have a smaller band gap $(1.6 \mathrm{eV}), \frac{57}{,}$, which, however, is related to a small polaron formation. Note that this value of the gap is smaller than ours $(1.724 \mathrm{eV})$ which does not include such polaronic effects. Whether or not such polaronic effects are observed may depend on the time scale of the method with which the gap is probed because the polaron formation occurs at the time scale of the atomic displacements. For example, one typically observes a polaron in photoluminescence but not in optical absorption. The present EELS measurements are closer to optical absorption.

In the present case of $\mathrm{Li}_{0.37} \mathrm{CoO}_{2}$ this reduction of the gap appears to be caused by the structural transitions, and changes of cation order. It is well known that disorder may reduce the band gap by forming defect like band tails in the gap near the band edges. However, in the present case, the situation may be more complex by the increasing importance of not only structural and cation disorder fluctuations but also magnetic moment formation and fluctuations in magnetic moment orientation and $d$-band correlation effects, including possibly strong electron-phonon coupling as occurs in self-trapped polaron formation. Disentangling these various effects is beyond the scope of the present paper.

\section{F. Conductivity}

Finally, we examine how the conductivity changes with annealing temperature and whether it is related to the observed band gap decrease and changes in valence, hybridization evidenced by the spectroscopic investigations of the previous sections. The conductivity changes as function of annealing temperature are shown in Fig. 9. The conductivity remains at its highest value for $T \leq 150^{\circ} \mathrm{C}$ corresponding to the $C 2 / \mathrm{m}$ phase. A decrease in conductivity is observed for $T \geq 150^{\circ} \mathrm{C}$. At $200^{\circ} \mathrm{C}$ it corresponds to a macroscopic $P 2 / m$ matrix, possibly with the beginning of $F d \overline{3} m$ nanodomains at $200^{\circ} \mathrm{C}$ (Supplementary Fig. S1(3)) $!^{39}$ This $F d \overline{3} m$ phase with its lower covalency and O-2 $p-$ Co- $3 d$ hybridization at the conduction band edges is, probably, at the origin of the start of conductivity decrease, as charge carriers tend to become more localized for less hybridized levels. This decrease is stronger for the macroscopic $F d \overline{3} m$ and $F m \overline{3} m$ phases at $250-350^{\circ} \mathrm{C}$. The spinel structure for $x=2 / 3$ was reported as insulating in STM when cation

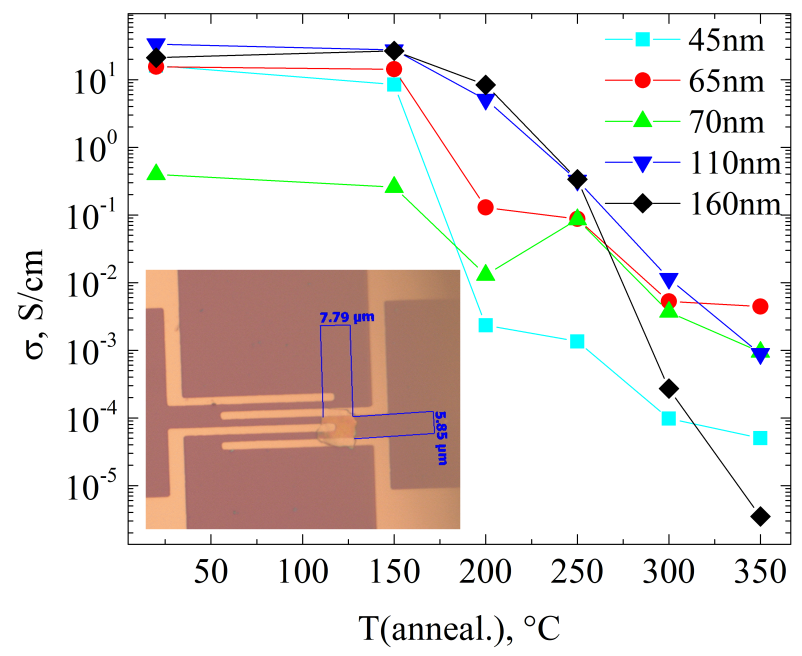

Fig. 9: Electrical conductivity as function of annealing temperature. Inset: device structure showing electrical contacts.

migration forces random occupation of sites $\frac{18}{18}$, In our case, the spinel type phase with $x \approx 1 / 3$ is also found to be insulating.

On the other hand, as mentioned earlier, there are also indications that on heating the $\mathrm{Li}$ concentration may be decreasing in the sample due to out-diffusion. The poorer the system is in $\mathrm{Li}$, the more correlated the electronic structure becomes with increasing polaronic effects 57 and this may also be part of the reason for the conductivity decrease. While for $\mathrm{Li}_{x} \mathrm{CoO}_{2}$ in the $R \overline{3} m$ and the closely related $C 2 / m$ and $P 2 / m$ structures, a normal band picture still holds with some $p$-type hole doping due to $x<1$, in the $F d \overline{3} m$ and even more so, in the $F m \overline{3} m$ phases, the starting picture of a band insulator becomes untenable because of the overlapping of the $t_{2 g}$ and $e_{g}$ bands. In the $F d \overline{3} m$ one would even have inverted $t_{2 g}$ and $e_{g}$ levels at tetrahedral Co-sites. The origin of the band gap in this case does no longer arise from a well separated nearly filled $t_{2 g}$ band below an empty $e_{g}$ band with low-spin configuration, but from correlation effects leading to magnetic moments and a gap forming between majority and minority spin electrons. The nature of the conductivity is thus clearly expected to be dramatically changing once these phases come into play. The inter-layer cation mixing might thus be considered to be detrimental for the conductivity.

\section{CONCLUSIONS}

To conclude, we have presented in this paper a comprehensive study of the structural changes, electronic properties as derived from electron energy loss spectroscopy correlated with first-principles calculations, and conductivity measurements of $\mathrm{Li}_{0.37} \mathrm{CoO}_{2}$ nanoflakes subjected to heating. The O- $K$ spectra were interpreted in terms of 
O- $p$ PDOS modulated by the dipole matrix elements linking the conduction states to the $\mathrm{O}-1 s$ core-wavefunction in an all-electron approach and taking the presence of the core-hole into account. The low energy loss spectra were well described by the calculated loss function $-\operatorname{Im}\left[\varepsilon^{-1}(\mathbf{q}, \omega)\right]$ for small $\mathbf{q}$. Predictions are made for the q-dependence of these spectra. They offer a basic interpretation of the main features, whose trends are studied upon heating.

The $\mathrm{Li}_{0.37} \mathrm{CoO}_{2}$ flakes are initially (between $150^{\circ} \mathrm{C}$ and $200^{\circ} \mathrm{C}$ annealing temperature) found to experience phase transitions from the rhombohedral $R \overline{3} \mathrm{~m}$ phase to the monoclinic phases $C 2 / m$ and $P 2 / m$ with disordered and ordered, Li vacancies respectively. During these transitions, the Co valence increases in parallel with increasing Co-3 $d-\mathrm{O}-2 p$ hybridization as evidenced from the interpretation of the peak ratio changes in $\mathrm{O}-K$ and $\mathrm{Co}-L_{2,3}$ spectra. Upon further heating above $250^{\circ} \mathrm{C}$ and completed by $350^{\circ} \mathrm{C}$ when partial or full Li-Co interlayer mixing happens in the spinel-type $F d \overline{3} m$ and rocksalttype $F m \overline{3} m$ phases, the Co nominal valency decreases, as well as the hybridization of $\mathrm{Co}-3 d-\mathrm{O}-2 p$ states. A band gap decrease is observed when these phases start to form. The increasing presence of $\mathrm{Co}^{2+}$ indicated by
$L_{2,3}$ spectra is consistent with a loss of Li from the rocksalt phase regions, as also indicated by the $\mathrm{O}-K$ spectra. These changes are related to the dramatically modified band structure which is no longer in a low-spin state. Band structure calculations at the GGA level indicate a significant overlap of $t_{2 g}$ and $e_{g}$ bands in the $F m \overline{3} m$ phase with a metallic band structure, which becomes unstable toward magnetic moment formation. The latter can be described within the DFT $+\mathrm{U}$ methodology and allowing the magnetic moments to occur in a disordered manner. This leads to a three to six orders of magnitude decrease in conductivity at temperatures where these phases start to form because of increased $\mathrm{Li}$ and Co interdiffusion forming a 3D network instead of a layered phase.

\section{ACKNOWLEDGMENTS}

This work was supported by the U.S. Air Force Office of Scientific Research under Grant No. FA9550-18-10030. The calculations made use of the High Performance Computing Resource in the Core Facility for Advanced Research Computing at Case Western Reserve University.
${ }^{1}$ K. S. Novoselov, A. K. Geim, S. V. Morozov, D. Jiang, Y. Zhang, S. V. Dubonos, I. V. Grigorieva, and A. A. Firsov, Science 306, 666 (2004).

2 S. Rahimi, L. Tao, S. F. Chowdhury, S. Park, A. Jouvray, S. Buttress, N. Rupesinghe, K. Teo, and D. Akinwande, ACS Nano 8, 10471 (2014)

3 K. F. Mak, C. Lee, J. Hone, J. Shan, and T. F. Heinz, Phys. Rev. Lett. 105, 136805 (2010)

4 B. Radisavljevic, A. Radenovic, J. Brivio, V. Giacometti, and A. Kis, Nature Nanotechnology 6, 147 (2011)

${ }^{5}$ L. Li, Y. Yu, G. J. Ye, Q. Ge, X. Ou, H. Wu, D. Feng, X. H. Chen, and Y. Zhang, Nature Nanotechnology 9, $372(2014)$

${ }^{6}$ P. Ares, F. Aguilar-Galindo, D. Rodr ÃAguez-San-Miguel, D. A. Aldave, S. DAaz-Tendero, M. AlcamA, F. MartAn, J. GAmez-Herrero, and F. Zamora, Advanced Materials 28, $6332(2016)$

T. Yang, T. T. Song, M. Callsen, J. Zhou, J. W. Chai, Y. P. Feng, S. J. Wang, and M. Yang, Advanced Materials Interfaces 6, 1801160 (2019)

${ }^{\delta}$ I. Altfeder, H. Lee, J. Hu, R. D. Naguy, A. Sehirlioglu, A. N. Reed, A. A. Voevodin, and C.-B. Eom, Phys. Rev. B 93, 115437 (2016)

${ }^{9}$ Z. Wang, Z. Zhong, X. Hao, S. Gerhold, B. Stöger, M. Schmid, J. Sánchez-Barriga, A. Varykhalov, C. Franchini, K. Held, and U. Diebold, Proceedings of the National Academy of Sciences 111, 3933 (2014), https://www.pnas.org/content/111/11/3933.full.pdf

10 S. Gonzalez, C. Mathieu, O. Copie, V. Feyer, C. M. Schneider, and N. Barrett, Applied Physics Letters 111, 181601 (2017)

i1 S. K. Radha and W. R. L. Lambrecht, "Spin-polarized two-dimensional electron/hole gases on $\mathrm{LiCoO}_{2}$ layers," (2020), arXiv:2003.00061 [cond-mat.str-el]

12 C. A. Marianetti, G. Kotliar, and G. Ceder, Nature Materials 3, 627 (2004)

is D.-L. Nguyen, C.-R. Hsing, and C.-M. Wei, Nanoscale 11, 17052 (2019)

${ }^{14}$ K. Takada, H. Sakurai, E. Takayama-Muromachi, F. Izumi, R. A. Dilanian, and T. Sasaki, Nature 422, $53(2003)$

${ }^{15}$ K. Iwaya, T. Ogawa, T. Minato, K. Miyoshi, J. Takeuchi, A. Kuwabara, H. Moriwake, Y. Kim, and T. Hitosugi, Phys. Rev. Lett. 111, 126104 (2013).

16 S. Sucharitakul, G. Ye, W. R. L. Lambrecht, C. Bhandari, A. Gross, R. He, H. Poelman, and X. P. A. Gao, ACS Applied Materials \& Interfaces 9, 23949 (2017)

${ }_{17}$ W.-B. Zhang, Q. Qu, and K. Lai, ACS Applied Materials \& Interfaces 9, 1702 (2017).

18 K. Miyoshi, K. Manami, R. Sasai, S. Nishigori, and J. Takeuchi, Phys. Rev. B 98, 195106 (2018).

19 L. Wu, W. H. Lee, and J. Zhang, Materials Today: Proceedings 1, 82 (2014), the 1st International Joint Mini-Symposium on Advanced Coatings between Indiana University-Purdue University Indianapolis and Changwon National University.

${ }^{20}$ K. G. Pachuta, E. B. Pentzer, and A. Sehirlioglu, Journal of the American Ceramic Society 102, 5603 (2019)

${ }^{21}$ K. G. Pachuta, E. B. Pentzer, and A. Sehirlioglu, Nanoscale Advances (2020), under review.

22 Y. Masuda, Y. Hamada, W. S. Seo, and K. Koumoto, Journal of Nanoscience and Nanotechnology 6, 1632 (2006)

${ }^{23}$ K. Crowley, K. Pachuta, S. K. Radha, H. Volkova, A. Se- 
hirlioglu, E. Pentzer, M.-H. Berger, W. R. L. Lambrecht, and X. P. A. Gao, The Journal of Physical Chemistry C xx, $\operatorname{xxx}(2020)$.

${ }_{24}$ https://jp-minerals.org/vesta/en/.

25 J. P. Perdew, K. Burke, and M. Ernzerhof, Phys. Rev. Lett. 77, 3865 (1996).

26 http://www .questaal .org

27 D. Pashov, S. Acharya, W. R. Lambrecht, J. Jackson, K. D. Belashchenko, A. Chantis, F. Jamet, and M. van Schilfgaarde, Computer Physics Communications 249, 107065 (2019)

28 G. Trimarchi, Z. Wang, and A. Zunger, Phys. Rev. B 97, $035107(2018)$

29 A. Zunger, S.-H. Wei, L. G. Ferreira, and J. E. Bernard, Phys. Rev. Lett. 65, 353 (1990).

30 Y. Zhang, J. Furness, R. Zhang, Z. Wang, A. Zunger, and J. Sun, Phys. Rev. B 102, 045112 (2020)

31 https://wiki.fysik.dtu.dk/gpaw/.

32 J. J. Mortensen, L. B. Hansen, and K. W. Jacobsen, Phys. Rev. B 71, 035109 (2005)

33 J. Enkovaara, C. Rostgaard, J. J. Mortensen, J. Chen, M. Dułak, L. Ferrighi, J. Gavnholt, C. Glinsvad, V. Haikola, H. A. Hansen, H. H. Kristoffersen, M. Kuisma, A. H. Larsen, L. Lehtovaara, M. Ljungberg, O. LopezAcevedo, P. G. Moses, J. Ojanen, T. Olsen, V. Petzold, N. A. Romero, J. Stausholm-Møller, M. Strange, G. A. Tritsaris, M. Vanin, M. Walter, B. Hammer, H. Häkkinen, G. K. H. Madsen, R. M. Nieminen, J. K. Nørskov, M. Puska, T. T. Rantala, J. Schiøtz, K. S. Thygesen, and K. W. Jacobsen, Journal of Physics: Condensed Matter 22, 253202 (2010)

${ }_{34}$ P. E. Blöchl, Phys. Rev. B 50, 17953 (1994)

35 S. L. Adler, Phys. Rev. 126, 413 (1962)

36 N. Wiser, Phys. Rev. 129, 62 (1963).

37 T. Motohashi, T. Ono, Y. Sugimoto, Y. Masubuchi, S. Kikkawa, R. Kanno, M. Karppinen, and H. Yamauchi, Phys. Rev. B 80, 165114 (2009).

38 K. Chang, B. Hallstedt, D. Music, J. Fischer, C. Ziebert, S. Ulrich, and H. J. Seifert, Calphad 41, 6 (2013),

39 Supplemental material contains HRTEM figures, full sets of EELS spectra in the O- $K$, Co- $L_{2,3}$ and low energy interband and plasmon ranges, calibration curve for Co valence, unit cell parameters for different phases and band structure figures.

${ }^{40}$ H. Kang, J. Lee, T. Rodgers, J.-H. Shim, and S. Lee, The Journal of Physical Chemistry C 123, 17703 (2019).

${ }^{41}$ Y. Shao-Horn, S. Levasseur, F. Weill, and C. Delmas, Journal of The Electrochemical Society 150, A366 (2003).

42 H. Wang, Y.-I. Jang, B. Huang, D. R. Sadoway, and Y.-M. Chiang, Journal of The Electrochemical Society 146, 473 (2019)

43 R. Gummow, D. Liles, and M. Thackeray, Materials Research Bulletin 28, 235 (1993)

44 S. Sharifi-Asl, F. A. Soto, A. Nie, Y. Yuan, H. AsayeshArdakani, T. Foroozan, V. Yurkiv, B. Song, F. Mashayek, R. F. Klie, K. Amine, J. Lu, P. B. Balbuena, and R. Shahbazian-Yassar, Nano Letters 17, 2165 (2017)

45 J. Kikkawa, S. Terada, A. Gunji, M. Haruta, T. Nagai, K. Kurashima, and K. Kimoto, Applied Physics Letters 104, 114105 (2014).

40 Y. Zhao, T. E. Feltes, J. R. Regalbuto, R. J. Meyer, and R. F. Klie, Journal of Applied Physics 108, 063704 (2010).

47 A. Savitzky and M. J. E. Golay, Analytical Chemistry 36, 1627 (1964).

48 https://materialsproject.org/

49 Z. Wang, J. Yin, and Y. Jiang, Micron 31, 571 (2000)

50 R. Egerton, Electron Energy-Loss Spectroscopy in the Electron Microscope (Springer US, Boston, MA, 2011).

51 C. Wolverton and A. Zunger, Phys. Rev. Lett. 81, 606 (1998)

52 M. J. Redman and E. G. Steward, Nature 193, 867 (1962)

53 J. Kikkawa, T. Mizoguchi, M. Arai, T. Nagai, and K. Kimoto, Phys. Rev. B 98, 075103 (2018)

54 J. M. Tarascon, C. Delacourt, A. S. Prakash, M. Morcrette, M. S. Hegde, C. Wurm, and C. Masquelier, Dalton Trans. , 2988 (2004).

55 K. Miedzinska, B. Hollebone, and J. Cook, Journal of Physics and Chemistry of Solids 48, 649 (1987)

50 J. van Elp, J. L. Wieland, H. Eskes, P. Kuiper, G. A. Sawatzky, F. M. F. de Groot, and T. S. Turner, Phys. Rev. B 44, 6090 (1991)

${ }^{7}$ T. J. Smart, T. A. Pham, Y. Ping, and T. Ogitsu, Phys. Rev. Materials 3, 102401 (2019). 